\title{
Direct numerical simulation analysis of local flow topology in a particle-laden turbulent channel flow
}

\author{
M. J. BIJLARD ${ }^{1}$, R. V. A. OLIEMANS ${ }^{1}$, L. M. PORTELA \\ AND G. OOMS $\mathrm{M}^{2} \dagger$ \\ ${ }^{1}$ J. M. Burgerscentrum, Delft University of Technology, Kramers Laboratorium, \\ Prins Bernhardlaan 6, 2628 BW Delft, The Netherlands \\ ${ }^{2}$ J. M. Burgerscentrum, Delft University of Technology, Laboratory for Aero- and \\ Hydrodynamics, Leeghwaterstraat 21, 2628 CB Delft, The Netherlands
}

(Received 26 May 2009; revised 5 January 2010; accepted 7 January 2010)

The results of point-particle Eulerian-Lagrangian direct numerical simulation (DNS) calculations of dilute particle-laden turbulent channel flow are used to study the effect of the particles on the local flow topology. It is found that in the viscous sublayer, the flow becomes increasingly more two-dimensional as the two-way coupling effect (due to interaction between particles and fluid flow) increases with increasing particle load. Beyond the viscous sublayer the modifications in flow topology are not strongly related to the preferential concentration of particles in the flow field, which is in contrast to previous channel flow simulations. The effect of particles on the turbulent flow beyond the viscous sublayer is mostly a result of the overall changing near-wall dynamics of the fluid flow.

\section{Introduction}

For several decades it has been known that adding solid particles to a turbulent fluid flow can result in a modification of the turbulence characteristics. To be able to isolate separate modulation effects the Euler-Lagrange approach with direct numerical simulations (DNS) or large-eddy simulations (LES) have been shown to be effective tools in the study of particle-laden flows. Several two-way coupled numerical studies have been focused on turbulence modulation in homogeneous, isotropic turbulence (see for instance Squires \& Eaton 1990; Elghobashi \& Truesdell 1993 and Boivin, Simonin \& Squires 1998), whereas others have been focused on turbulent homogeneous shear flow (see Ahmed \& Elghobashi 2000).

For fully developed particle-laden wall-bounded flows a fairly limited amount of studies have been reported on turbulence modulation effects using DNS or LES results. Yamamoto et al. (2001) carried out an LES study for a particle-laden flow in a channel. A similar study was performed by Li et al. (2001), who made twoway coupled DNS calculations for a channel flow, but at a much lower Reynolds number and finer resolution with also considerably smaller particle inertia than that of Yamamoto et al. (2001). 
The objectives of our study are to perform high-resolution Euler-Lagrange DNS calculations for a turbulent particle-laden flow in a two-dimensional channel and to use the results to improve our understanding of the physics of particle-turbulence interaction in wall-bounded flows. In this publication we will focus on the change in local flow topology due to the presence of the particles. A unique description of local flow patterns was introduced by Chong, Perry \& Cantwell (1990). Their general classification scheme, which is based on invariants of a smooth three-dimensional vector field, provides a method to study the full range of turbulent scales. With this scheme it is possible to combine local flow patterns in physical space with their structural properties in the space of invariants. Since Chong et al. this topology invariant technique has further matured and became, apart from visualization purposes, a popular tool to investigate many fundamental questions in turbulence. So far various flow conditions have been studied using this technique, for instance, isotropic turbulence (Ooi et al. 1999), free shear flows (Soria \& Cantwell 1994; Soria, Ooi \& Chong 1997), vortex shedding (Perry \& Chong 1994), time-developing mixing layers (Soria et al. 1994), turbulent boundary layers (Chacín, Cantwell \& Kline 1996; Chacín \& Cantwell 2000) and turbulent channel flow (Blackburn, Mansour \& Cantwell 1996). Even though the large-scale characteristics among these studies are totally different, it turned out that most topology invariant plots of the velocity fields have a similar tear-drop shape. This universal character appears however to diminish in the direct vicinity of a no-slip wall (this holds particularly in the viscous sublayer, see Blackburn et al. 1996).

Applications of the local topology method to a turbulent particle-laden flow were introduced by Rouson \& Eaton (2001). In their one-way coupled turbulent channel flow study, the approach of Blackburn et al. was chosen to support the idea that particle segregation should have some preference for specific kinds of coherent flow structures. Light particles showed a tendency to avoid the strongest vortical, and vortex-stretching regions, whereas the larger inertia particles did not show a strong preference for any of the topological regions. This effect was most pronounced in the viscous sublayer and became weaker with increasing distance from the wall. More recently, a similar kind of study was performed by Picciotto, Marchioli \& Soldati (2005). Instead of analysing the non-randomness of the particles throughout the entire channel width, their study focused on smaller inertia particles and used this topology analysis as a kind of objective measure to improve the understanding of short- and long-term particle accumulation processes in the near-wall region.

Our DNS calculations differ from earlier ones by considering a significantly higher spatial resolution, a larger streamwise channel length and larger mass loadings. We restrict ourselves to point particles and ignore gravity effects. In our simulations the mass fraction ranges between $\phi_{m}=0$ and 0.65 , the Kolmogorov-based Stokes number $(S t)$ ranges between 1 and 7 and the dimensionless wall-unit scaled particle relaxation time ranges between 14 and 111. Figure 1 shows the span of our simulations in the Elghobashi turbulence modulation map (Elghobashi 1994) with St, particle volume fraction and length ratios as parameters on the axes. According to this map our simulations are globally dilute flows in the heart of the region with two-way coupling.

\section{Details of DNS calculations}

The total volume fraction of the particles is assumed to be always much smaller than the considered control volume in the channel. In order to track the particles in the flow field the Lagrangian trajectory of each individual particle is calculated. 


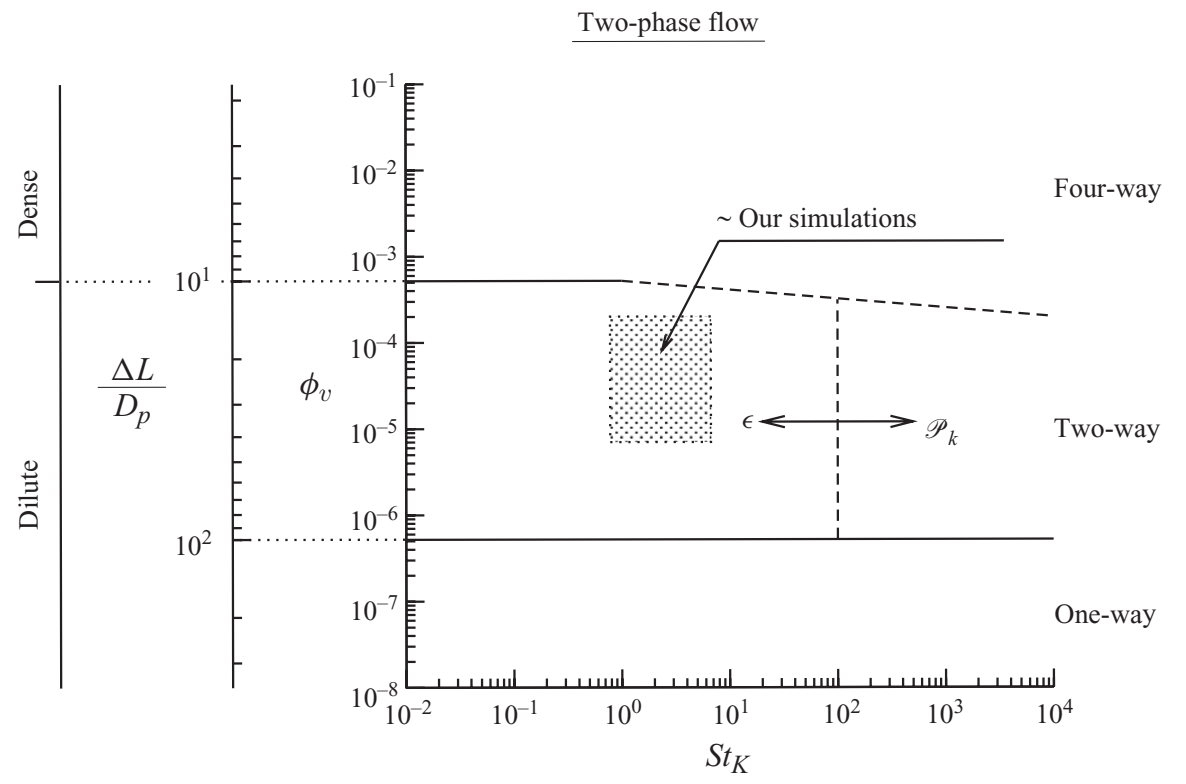

FiguRE 1. Turbulence modification; based on Elghobashi (1994). $\Delta L$ is the size of a grid cell, $D_{p}$ is the particle diameter and $S t_{K}$ is the Stokes number based on the Kolmogorov length scale.

The equation of motion for a particle is complex, because it depends on several forces acting on it. From a numerical point of view it is rather expensive to compute the complete forcing term for each individual particle, particularly because millions of particles are considered. Therefore, we assume in our calculations that the ratio between particle density and fluid density is very large. In that case the particle trajectories are mainly determined by Stokes drag,

$$
F_{i}\left(x_{j}^{(n)}\right) \simeq 3 \pi \mu D_{p}\left(U_{i}^{(p)}-V_{i}\right),
$$

where $F_{i}\left(x_{j}^{(n)}\right)$ is the force acting on the $n$th particle, $x_{j}^{(n)}$ is the spatial coordinate of the $n$th particle, $\mu$ is the dynamic viscosity, $D_{p}$ is the particle diameter, $U_{i}^{(p)}$ is the fluid velocity at the position of the $n$th particle and $V_{i}$ is the particle velocity. As the particles are assumed to be significantly smaller than the smallest flow scale, the particle feedback force $F_{i}$ is modelled using a simple point-particle approach. (We will come back to this point in more detail.)

A standard finite-volume single-phase solver with a semi-implicit coupling scheme is used to solve the incompressible Navier-Stokes equations for the continuous phase. The numerical method of the flow solver is essentially based on a twostep predictor-corrector approach. First, an explicit second-order Adams-Bashforth method is used for the time advancement of both the convective and diffusion terms, where the discretized time-step is determined by a standard Courant stability criterion. This part also includes the driving force to maintain the flow, i.e. the fixed, external pressure gradient in the streamwise direction. After this provisional velocity distribution is computed, a corrector step is enforced through the continuity equation. It numerically solves the Poisson equation for the pressure, using a Fourier series expansion in the homogeneous directions, together with tridiagonal matrix inversion for the inhomogeneous direction. 


\begin{tabular}{ccccccccc}
\hline Grid ID & $R e_{\tau}$ & $L_{x}$ & $L_{y}$ & $L_{z}$ & $N_{x} \times N_{y} \times N_{z}$ & $\Delta x^{+}$ & $\Delta y^{+}$ & $\Delta z^{+}$ \\
3a & 180 & $5 H$ & $2 H$ & $H$ & $192 \times 128 \times 96$ & 9.4 & 5.6 & $0.6-4.8$ \\
3b & 180 & $5 H$ & $2 H$ & $H$ & $192 \times 192 \times 170$ & 9.4 & 3.8 & $0.5-3.8$ \\
3c & 180 & $10 H$ & $2 H$ & $H$ & $384 \times 128 \times 96$ & 9.4 & 5.6 & $0.6-4.8$ \\
$5 \mathrm{a}$ & 250 & $5 H$ & $2 H$ & $H$ & $256 \times 192 \times 128$ & 9.8 & 5.2 & $1.1-5.1$ \\
$5 \mathrm{~b}$ & 250 & $10 H$ & $2 H$ & $H$ & $512 \times 192 \times 128$ & 9.8 & 5.2 & $1.1-5.1$ \\
& \multicolumn{7}{c}{ TABLE 1 1. Mesh specifications. }
\end{tabular}

As two-way coupling is considered, the continuous phase solver needs some modification to incorporate the additional forces between the dispersed phase and the carrier fluid. Our numerical implementation of this two-way coupling mechanism is based on a straightforward explicit method, which works according to three basic steps: first, the predictor part of the continuous phase solver is computed to predict the fluid acceleration value without the particle forcing term, $F_{i}$. Secondly, an update of this fluid acceleration value is carried out through incorporating the particle force contributions, yielding a provisional update of $U_{i}$. Finally, the provisional velocity value $U_{i}$ is corrected, such that it enforces the continuity equation. So the only difference to the unladen, or one-way coupled, simulations is formed by the second step.

The numerical simulations share the following characteristics. For our particleladen simulations the motion of each particle is calculated using the aforementioned Lagrangian tracking method, wherein only linear Stokes drag and elastic bouncing at the wall are considered. All particles are mono-sized $\left(D_{p}=10^{-3} \mathrm{H}\right)$; they remain always smaller than the smallest grid-cell dimension. $H$ is the height of the channel. The results are for two modest low Reynolds numbers: $R e_{\tau}=180$ and $R e_{\tau}=250$. These Reynolds numbers are based on the friction velocity $u_{\tau}$ and the half channel height $H / 2$. For both Reynolds numbers a similar channel geometry is used: a streamwise channel length of either $L_{x}=5 \mathrm{H}$ or $L_{x}=10 \mathrm{H}$ with a spanwise width $L_{y}=2 H$. In the streamwise $(x)$ and spanwise $(y)$ directions we use periodic boundary conditions on a uniform grid, while for the normal $(z)$ direction no-slip boundary conditions and a non-uniform grid stretching are used to resolve the smallest flow scales near the walls. Furthermore, to keep the total amount of particles constant during a simulation, the particles are re-introduced, i.e. if they leave the computational domain, either in the streamwise or spanwise direction, the particles are fed back in with the same velocity at the opposite side.

For our simulations an overview of the flow geometries and grid resolutions is given in table 1 . The computational domain lengths $L_{x}, L_{y}, L_{z}$ correspond to the streamwise $(x)$, spanwise $(y)$ and wall-normal $(z)$ directions, respectively. The amount of grid points along the three directions are represented by $N_{x}, N_{y}, N_{z}$, with corresponding grid resolutions $\Delta x^{+}, \Delta y^{+}, \Delta z^{+}$, given in wall units. A wall unit of length and time are defined by $z^{+}=\left(z u_{\tau}\right) / v$ and $t^{+}=\left(t u_{\tau}^{2}\right) / v$, respectively. 'Short' and 'long' streamwise channel domains are considered. This channel enlargement is crucial for higher particle mass loading simulations. Obviously, longer streamwise simulations are computationally more expensive.

Our simulation parameters are summarized in table 2. A division can be made between the presented parameters: parameters related to the global particle mass loading and parameters related to the particle dynamics. The parameters related to mass loading $\rho_{p}, D_{p}^{+}, N_{p}, \phi_{m}$ and $\phi_{v}$, denote respectively, the particle density 


\begin{tabular}{cccccccccc}
\hline Run ID & Grid ID & $R e_{\tau}$ & $\rho_{p}$ & $D_{p}^{+}$ & $N_{p}$ & $\phi_{m}$ & $\phi_{v}$ & $S t^{+}$ & $S t_{K}$ \\
R3.0 & 3a-b & 180 & - & - & - & - & - & - & - \\
R3.1 & 3c & 180 & 2000 & 0.36 & 3.161 .088 & 0.16 & $8.28 \times 10^{-5}$ & 14 & 0.96 \\
R3.2 & 3c & 180 & 2000 & 0.36 & 12.644 .352 & 0.65 & $3.31 \times 10^{-4}$ & 14 & 0.79 \\
O3.3 & 3a & 180 & 8000 & 0.36 & 1.580 .544 & 0.64 & $8.28 \times 10^{-5}$ & 58 & 4.0 \\
R3.3 & 3a-b & 180 & 8000 & 0.36 & 393.260 & 0.16 & $2.06 \times 10^{-5}$ & 58 & 3.9 \\
R3.4 & 3c & 180 & 8000 & 0.36 & 3.161 .088 & 0.65 & $8.28 \times 10^{-5}$ & 58 & 3.2 \\
R5.0 & 5a & 250 & - & - & - & - & - & - & - \\
R5.1 & 5a & 250 & 2000 & 0.5 & 1.577 .961 & 0.16 & $8.26 \times 10^{-5}$ & 28 & 1.5 \\
O5.2 & 5a & 250 & 8000 & 0.5 & 395.352 & 0.16 & $2.07 \times 10^{-5}$ & 111 & 6.7 \\
R5.2 & 5a & 250 & 8000 & 0.5 & 395.352 & 0.16 & $2.07 \times 10^{-5}$ & 111 & 6.1 \\
R5.3 & 5b & 250 & 8000 & 0.5 & 3.155 .922 & 0.65 & $8.26 \times 10^{-5}$ & 111 & 4.8
\end{tabular}

TABLE 2. Simulation parameters. 'One-way' coupled simulations are denoted by the 'prefix' O.

(normalized by the gas density), the diameter of the particle (normalized by wall units), the total number of particles in the system, the total mass-fraction of the dispersed phase and the total volume fraction of the particles. As can be noticed, the total number of particles within the system may become quite large (up to almost 13 million), but their bulk volume fractions remain strictly within the dilute regime $\left(\phi_{v} \lesssim 10^{-3}\right)$. The second group of parameters, shown in table 2 , represents two Stokes numbers $\left(S t^{+}\right.$and $\left.S t_{K}\right)$. Generally, the non-dimensional $S t$ is given by $S t=\tau_{p} / \tau_{f}$ : the ratio of the relaxation time of a particle and the relaxation time of the surrounding fluid. For $\rho_{p} \gg 1$, the particle relaxation time $\left(\tau_{p}\right)$ can be expressed in the Stokes limit as $\tau_{p}=\rho_{p} D_{p}^{2} /(18 v)$, while for the characteristic time scale of the fluid $\left(\tau_{f}\right)$ several measures can be chosen. In the extremes, such as very light or very heavy particles, the particle motion behaves (approximately) as a fluid element or as a ballistic projectile. Our particles possess 'intermediate' inertias, and thus lay somewhere between these two extremes. It should be noted that these 'intermediate' particle inertias are of great interest, mainly because they are known to show strong preferential concentrations. In $S t^{+}$a time scale is used based on wall units; in $S t_{K}$ the Kolmogorov time scale is used.

In figure 2 a sketch is shown for the distribution of the grid points (indicated by crosses) near the wall for two cases $\left(N_{z}=96\right.$ and $\left.N_{z}=170\right)$. The horizontal coordinate of the crosses gives the distance of the grid points to the wall in wall units and their vertical coordinate the grid-stretching increments. Also, the dimensionless Kolmogorov length scale $\left(\eta_{K}^{+}\right)$in the near wall region is shown as function of the distance to the wall (by the solid line); its value is given along the vertical axis. The size of the particles is given by the diameter of the black circle (expressed in wall units as indicated by the horizontal axis). As can be seen the size of the particles $\left(0.36^{+}\right)$ is considerably smaller than the Kolmogorov length scale $\left(\sim 1.5^{+}\right)$and also smaller than the size of the grid elements $\left(0.5^{+}-2.0^{+}\right)$(dependent on the value of $N_{z}$ ).

There is a point of concern in this type of simulation concerning accuracy. Several two-way coupling numerical studies using the point-particle assumption in homogeneous, isotropic turbulence have shown that this method can be used with confidence if some specific conditions are satisfied. In particular, the particle diameter must remain much smaller than the smallest energetic turbulent length scales and smaller than the size of the control volume, appreciably. These criteria can be satisfied quite easily in unbounded flow configurations such as homogeneous turbulence or 


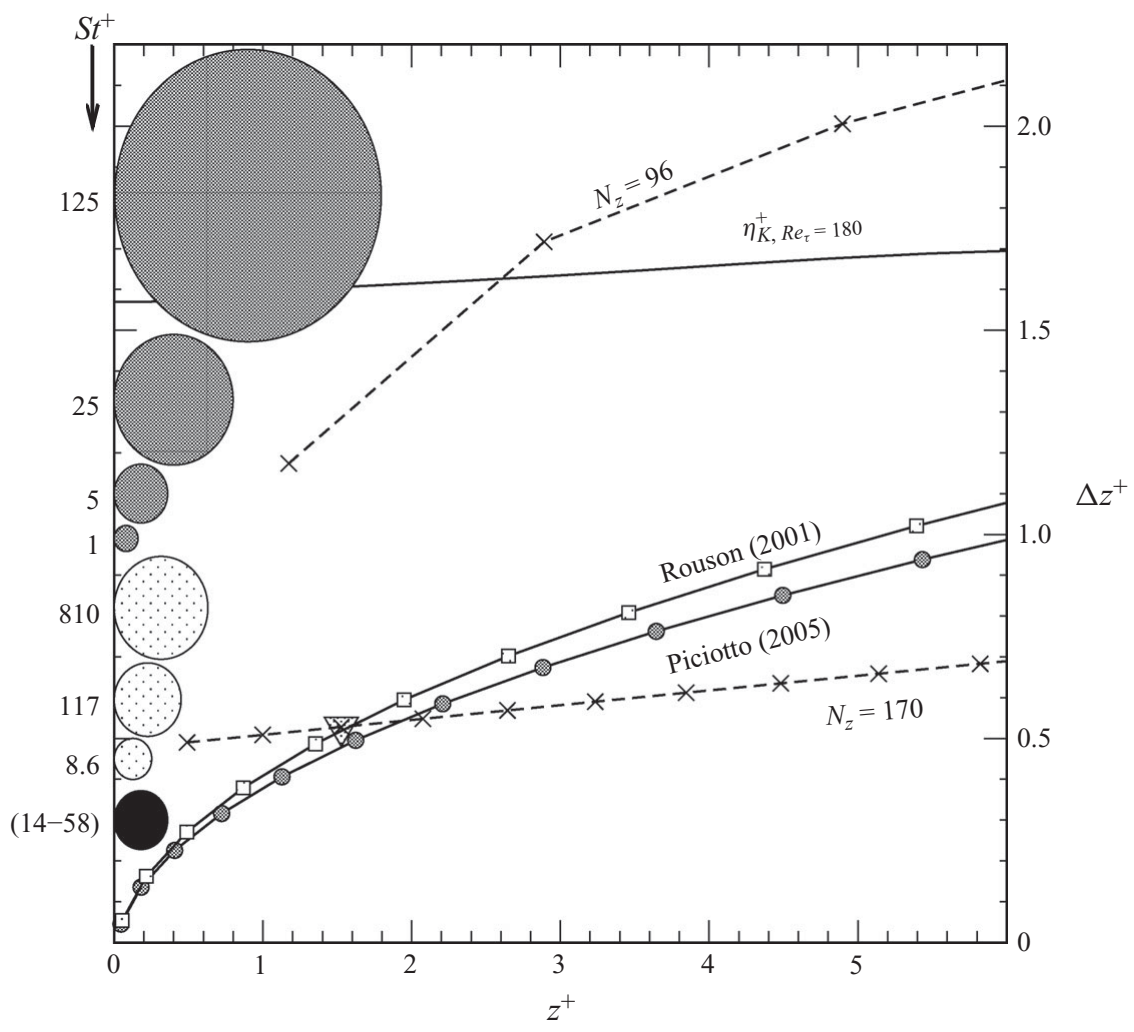

FIGURE 2. Consequence of finite-sized particle 'diameters' on several wall-normal grid resolutions in the vicinity of the wall $\left(0 \leqslant z^{+}<6\right)$; here the crosses denote the actual wall-normal grid locations, whereas the vertical axis displays the corresponding interspatial grid-stretching increments. Particle wall-touching locations of three different particle-laden studies: black-coloured particle displays the particle diameter of our low-Reynolds-number simulations $\left(R e_{\tau}=180 \rightarrow D_{p}^{+}=0.36\right)$; three light grey particles depict the diameters studied in Rouson \& Eaton (2001) $\left(D_{p}^{+}=[0.25 / 0.45 / 0.63]\right)$; four dark grey particles approximately correspond with the results of Picciotto et al. (2005) and Soldati $(2005)\left(D_{p}^{+} \approx[0.16 / 0.36 /\right.$ $0.8 / 1.79])$.

free-jet turbulence, but are conditions difficult to satisfy in wall-turbulent flows. Considering the second criterion we can notice that the diameter of the particles in our study can be of the same order as or considerably smaller than the near-wall grid size dependent on the value of $N_{z}$ (see figure 2). Satisfying the first condition should ensure that the very local fluid deformation induced by the presence of the particles can be neglected in the two-way coupling effect. Although the particles in our study are smaller than the Kolmogorov length scale, this criterion remains difficult to satisfy in the near-wall region as small riblets of particles can be formed that can drastically change the flow behaviour. The importance of this point could be checked by comparing our results with the results of DNS for finite-sized particles. However, these studies (see Uhlmann 2008 and references therein) concentrate on the two-way coupling effect of a relatively small number of relatively large particles $\left(N_{p} \approx 10-1000\right.$, $\left.D_{p}^{+} \approx 10-30\right)$ considering also the effect of gravity. Due to the particle diameter not only the interaction with the turbulent flow structures exists, but also the influence of 
the particle wakes plays an important role. This wake effect is absent in our study. So a comparison of these studies with our work is not straightforward.

Our code was part of an international collaborative benchmark test concerning direct numerical simulations of particle dispersion in wall-bounded turbulence without two-way coupling effect (see Marchioli et al. 2008). The objective was to establish a source of data relevant for this phenomenon. Different numerical approaches and computational codes were used to simulate the particle-laden flow and calculations were carried on long enough to achieve a statistically steady condition for the particle distribution. A comprehensive database including both post-processed statistics and raw data for the fluid and the particles was obtained. Direct comparison of the statistics allowed an observation of how well different codes performed when applied to the same problem and how the accuracy of the results depended on the choice made in terms of simulation parameter values. The results of our code were in agreement with those of other codes.

\section{Local flow topology}

The topology of the flow at a certain point in the flow field can be studied by analysing the following equation:

$$
\dot{x}_{i}=A_{i j} x_{j}
$$

where the vector $\boldsymbol{x}$ represents the distance to the considered point in the flow field and $A_{i j}$ represents the rate-of-deformation tensor, which can be divided into a symmetric and an antisymmetric part

$$
A_{i j}=\frac{\partial U_{i}}{\partial x_{j}}=\frac{1}{2}\left(\frac{\partial U_{i}}{\partial x_{j}}+\frac{\partial U_{j}}{\partial x_{i}}\right)+\frac{1}{2}\left(\frac{\partial U_{i}}{\partial x_{j}}-\frac{\partial U_{j}}{\partial x_{i}}\right)=S_{i j}-\frac{1}{2} \varepsilon_{i j k} \Omega_{k} .
$$

Setting $x_{i}=C_{i} \exp \left(\lambda_{\alpha} t\right)$ in (3.1) the usual eigenvalue problem is derived, reading

$$
\operatorname{det}\left(A_{i j}-\lambda_{\alpha} \delta_{i j}\right)=0,
$$

which yields the cubic characteristic equation for the second-order tensor $A_{i j}$

$$
\lambda_{\alpha}^{3}+P_{A} \lambda_{\alpha}^{2}+Q_{A} \lambda_{\alpha}+R_{A}=0
$$

where $P_{A}, Q_{A}$ and $R_{A}$ are the tensor invariants. Under incompressible flow conditions the behaviour of (3.1) is governed by these three different invariants (instead of the nine $A_{i j}$ components). As function of the three corresponding eigenvalues $\lambda_{\alpha}$ the invariants are given by

$$
\left.\begin{array}{l}
P_{A}=-\left(\lambda_{1}+\lambda_{2}+\lambda_{3}\right)=0 \\
Q_{A}=\left(\lambda_{1} \lambda_{2}+\lambda_{2} \lambda_{3}+\lambda_{3} \lambda_{1}\right)=-\frac{1}{2}\left(\lambda_{1}^{2}+\lambda_{2}^{2}+\lambda_{3}^{2}\right), \\
R_{A}=-\left(\lambda_{1} \lambda_{2} \lambda_{3}\right)=-\frac{1}{3}\left(\lambda_{1}^{3}+\lambda_{2}^{3}+\lambda_{3}^{3}\right),
\end{array}\right\}
$$

where the latter alternative expressions of $Q_{A}$ and $R_{A}$ in (3.5) are simply a result of substituting the incompressibility constraint of $P_{A}$. So the topological classification in $P_{A}-Q_{A}-R_{A}$ space is reduced to a classification in a two-dimensional $Q_{A}-R_{A}$ plane.

Since the characteristic equation (3.4) is cubic and the tensor invariants are real, at least one eigenvalue is real whereas the other two may be either real or form a complex conjugate pair (note, when an explicit distinction is made between the 


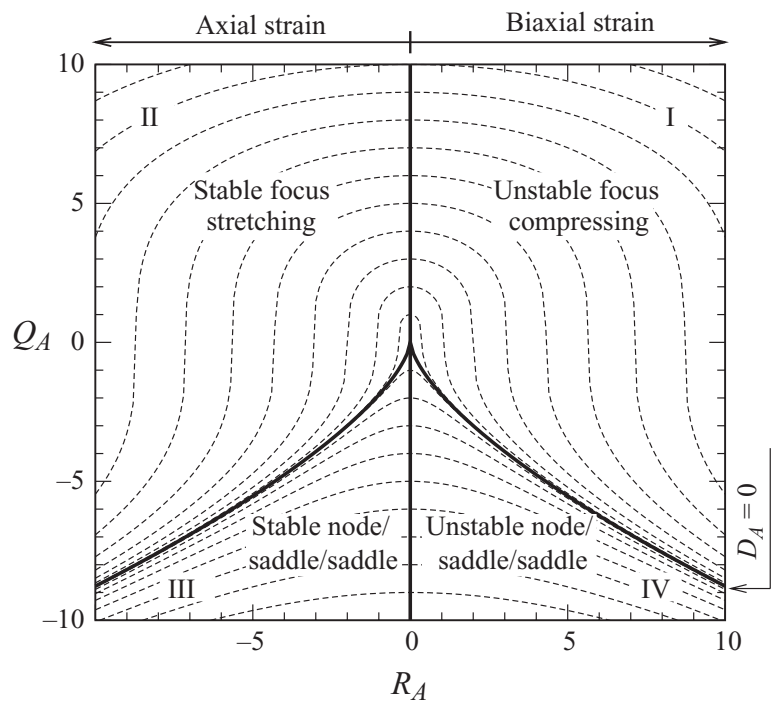

FIGURE 3. Classification of topology characteristics for tensor invariants $Q_{A}$ and $R_{A}$, restricted to incompressible flow conditions (see Chong et al. 1990). Dashed lines correspond to different $D_{A}$-isolines.

three eigenvalues, we shall consistently present their real parts always in a descending order: $\left.\operatorname{Re}\left(\lambda_{1}\right) \geqslant \operatorname{Re}\left(\lambda_{2}\right) \geqslant \operatorname{Re}\left(\lambda_{3}\right)\right)$. The sign of the real part of the corresponding eigenvalues determines the behaviour of the local flow pattern: $\operatorname{Re}\left(\lambda_{\alpha}\right)<0$ denotes a stable, positive attractor, while $\operatorname{Re}\left(\lambda_{\alpha}\right)>0$ corresponds to an unstable, negative attractor. When two eigenvalues are complex conjugates (with pure imaginary parts $\left.\operatorname{Im}\left(\lambda_{i}, \lambda_{j}\right)= \pm \mathrm{i} \omega\right)$, the flow pattern corresponds to local circular orbits, which are perpendicularly orientated to the flow pattern corresponding to the third pure real eigenvalue. For incompressible flow conditions this distinction between real and partly imaginary eigenvalues corresponds to a mapping of two distinct subregions in the $Q_{A}-R_{A}$ phase plane: all roots of (3.4) are real if the discriminant $D_{A}<(27 / 4) R_{A}^{2}+Q_{A}^{3}$, while for $D_{A}>(27 / 4) R_{A}^{2}+Q_{A}^{3}$ two roots form a complex conjugate pair. Furthermore, since only two of the three eigenvalues are independent (the third one is the sum of the other two, because $P_{A}=0$ ), the three-dimensional topology becomes characterized by either axial strain $\left(R_{A}<0\right.$; real part: $\left.\lambda_{1}>0, \lambda_{2}, \lambda_{3}<0\right)$ or biaxial strain $\left(R_{A}>0\right.$; real part: $\left.\lambda_{1}, \lambda_{2}>0, \lambda_{3}<0\right)$. The different flow topologies are summarized in figure 3 . The four topological quadrants (denoted by I-IV) are subdivided by the tent-like curve $D_{A}=0$ and the vertical axis $R_{A}=0$ : (I) unstable focus/compressing $\left(\lambda_{1,2}=(\lambda / 2) \pm \mathrm{i} \omega\right.$ and $\left.\lambda_{3}=-\lambda<0\right)$; (II) stable focus/stretching $\left(\lambda_{1}=\lambda>0\right.$ and $\left.\lambda_{2,3}=-(\lambda / 2) \pm \mathrm{i} \omega\right)$; (III) stable node/saddle/saddle $\left(\lambda_{1}>0\right.$ and $\left.\lambda_{2,3}<0\right)$; (IV) unstable node/saddle/saddle $\left(\lambda_{1,2}>0\right.$ and $\left.\lambda_{3}<0\right)$.

Instead of presenting the invariants as a function of their corresponding eigenvalues, the invariants of $A_{i j}$ may also be expressed according to the more familiar turbulence quantities, namely, $S_{i j}$ and $\Omega_{i}$ :

$$
\left.\begin{array}{l}
P_{A}=-A_{i i}=-S_{i i}=0, \\
Q_{A}=-\frac{1}{2} A_{i j} A_{j i}=-\frac{1}{2}\left(S_{i j} S_{j i}-\frac{1}{2} \Omega_{i}^{2}\right)=Q_{s}+Q_{W}, \\
R_{A}=-\operatorname{det}\left(A_{i j}\right)=-\frac{1}{3} A_{i j} A_{j k} A_{k i}=-\frac{1}{3}\left(S_{i j} S_{j k} S_{k i}+\frac{3}{4} \Omega_{i} \Omega_{j} S_{j i}\right)=R_{S}+R_{W},
\end{array}\right\}
$$


which shows that the second invariant $Q_{A}$ consists of the difference between strain and vorticity contributions. The strain contribution of $Q_{A}$, represented by $Q_{S}=-\left(S_{i j} S_{j i} / 2\right) \leqslant 0$, is directly proportional to the total viscous dissipation $\left(=2 v S_{i j} S_{i j}\right)$, whereas the remaining $Q_{W}=\left(\Omega_{i}^{2} / 4\right) \geqslant 0$ contribution is proportional to the total enstrophy $\left(=v \Omega_{i}^{2}\right)$. Thus, large positive (negative) values of $Q_{A}$ indicate flow regions where enstrophy is large (small) compared to viscous dissipation. The third invariant $R_{A}$ also consists of two contributions. However, the pure strain contribution $R_{s}=-\left(S_{i j} S_{j k} S_{k i}\right) / 3$ does not directly resemble a common turbulence quantity, while instead the second part $R_{W}=-\left(\Omega_{i} \Omega_{j} S_{j i} / 4\right)$ corresponds to the total generation of enstrophy by vortex stretching.

The invariants of the velocity-gradient tensor $A_{i j}$ consist of first-order spatial derivative products of the instantaneous velocity fields, meaning that the typical length scales of $\partial_{x_{i}}$ cover a range between the characteristic length scale of the mean flow $(\mathscr{L})$ and the dissipative Taylor length scale $\left(\lambda_{T}\right)$, i.e. $\partial_{x_{i}} \propto\left[\mathscr{L}^{-1}, \lambda_{T}^{-1}\right]$. The largest length scales will generally contribute little to the invariants of $A_{i j}$, whereas the smaller dissipative scales contribute, on average, the most to the intensities of $Q_{A}$ and $R_{A}$. Using classical scaling arguments (see Tennekes \& Lumley 1972), it can readily be shown that the ratio between the large length scale and Taylor length scale increases progressively with Reynolds number $\left(\lambda_{T} / \mathscr{L} \propto R e_{m}^{-1 / 2}\right)$. Hence the intensities of $Q_{A}$ and $R_{A}$ scale accordingly: $A_{i j} A_{j i} \sim R e_{m}$ and $A_{i j} A_{j k} A_{k i} \sim R e_{m}^{3 / 2}$.

\section{Results}

For a unidirectional flow like our channel flow, the turbulence activity and flow topology vary as function of the inhomogeneous direction. Hence, the visualization space of the flow topology has three dimensions: one for $Q_{A}$, one for $R_{A}$ and one for the distance to the wall. Since such representations are generally difficult to analyse, we reduce (as was done by Blackburn et al. 1996) the dependence on the distance to the wall of the joint p.d.f.s for $Q_{A}, R_{A}$ to four classes by averaging over four wall-parallel two-dimensional regions. These are the usual four regions: the viscous sublayer $\left(0<z^{+} \leqslant 5\right)$, the buffer layer $\left(5<z^{+} \leqslant 35\right)$, the 'log' region $\left(35<z^{+} \leqslant 150\right)$ and the outer region $\left(150<z^{+} \leqslant R e_{\tau}\right)$. Despite this significant reduction, the corresponding number of p.d.f.s is still quite substantial. The reason is that for each test case four wall-parallel volume averages are sampled at the grid points and at the in- and outward migrating particle locations (clarifying if their possible distinct characters are noticeable in the invariant space of $Q_{A}, R_{A}$ ). Therefore, we shall limit ourselves here to the evaluation of the joint p.d.f.s as functions of mass loading and of particle relaxation time. Many more results can be found in Bijlard (2009).

In order to present reasonably smooth and consistent joint p.d.f.s of $Q_{A}, R_{A}$, we calculated the invariant distributions by taking the whole ensemble average of all uncorrelated flow fields of each test case. Moreover, we considered only those test cases which have been performed for the same grid resolution. This resolution, given by $\left(N_{x} \times N_{y} \times N_{z}\right)$, is $192 \times 128 \times 96$ (for the 'long' streamwise channels $N_{x}$ is twice as large, though $\Delta x$ remains unchanged). Thus every component of the velocity gradient tensor is calculated at each interior grid point, which then is used to determine the local $Q_{A}, R_{A}$ values of the flow at the grid points and/or at the particle locations through trilinear interpolation. Thereafter, the results get progressively smoothed by the ensemble averaging procedure.

\subsection{Viscous sublayer (from an invariant perspective)}

Figure 4 shows the volume-averaged joint p.d.f.s for $Q_{A}, R_{A}$ in the viscous sublayer as a function of the mass loading. First, the joint $Q_{A}, R_{A}$ distribution of the one-way 

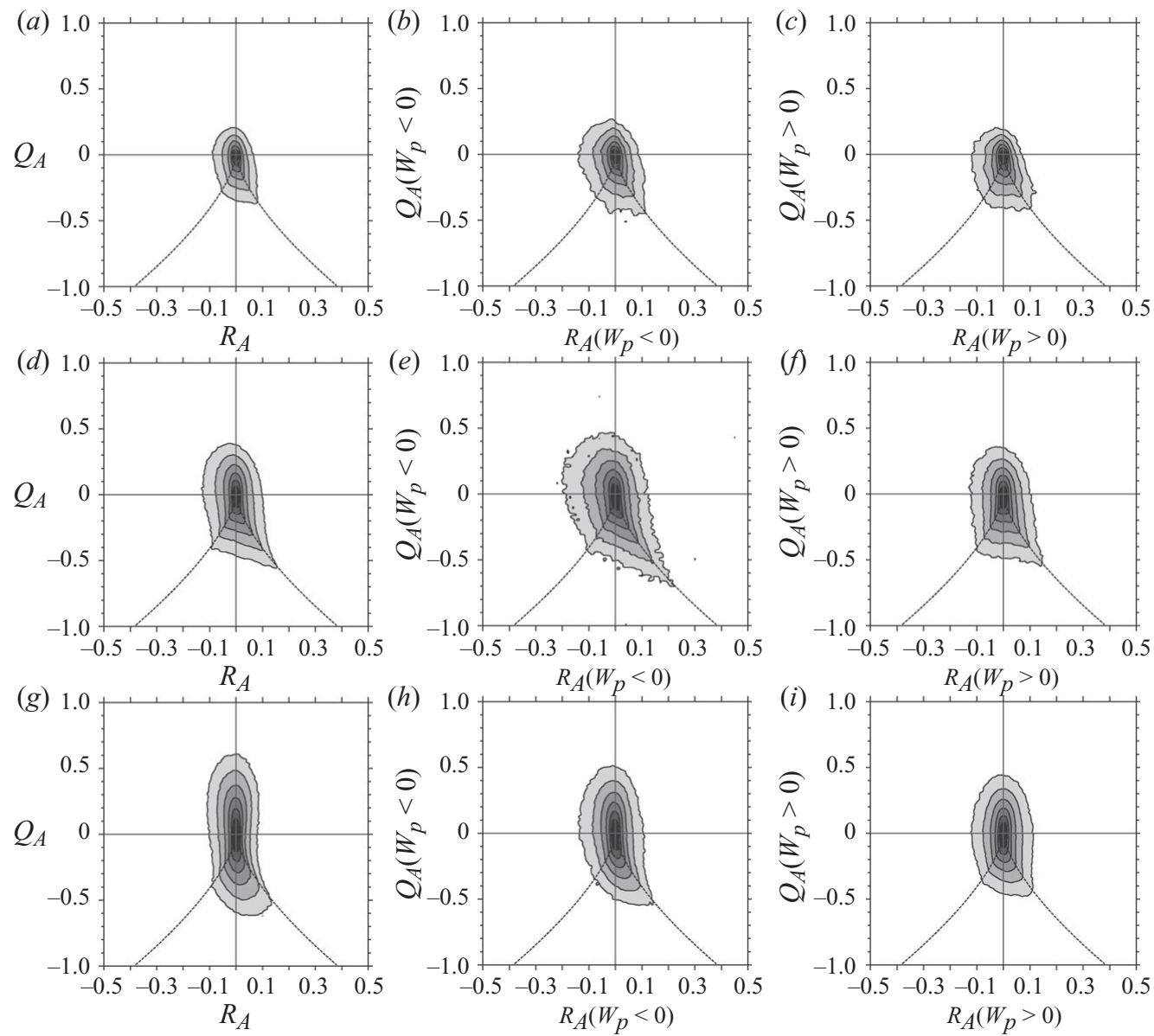

FIGURE 4. Effect of mass loading on normalized joint p.d.f. of $Q_{A}, R_{A}$ in the viscous sublayer $\left(0<z^{+} \leqslant 5\right)$ for the $R e_{\tau}=180$ case with $\tau_{p}^{+} \simeq 58$. $(a-c)$ O3.3: one-way coupling; $(d-f)$ R3.3: $\phi_{m} \simeq 0.16 ;(g-i)$ R3.4: $\phi_{m} \simeq 0.65$. $(a),(d)$ and $(g)$ : flow field sampled at grid locations. Flow field conditionally sampled at particle locations, respectively, $(b),(e)$ and $(h)$ : at inward (to the wall) migrating particle locations and $(c),(f)$ and $(i)$ : outward (to the channel centre) migrating particle locations. The grey scales denote a logarithmic decade, ranging over six decades.

coupling simulations, sampled at the grid locations, is considered (figure $4 a$ ). One can notice (in agreement with other studies) that the larger excursions of the joint p.d.f.s have a higher probability to be located in either the stable focus/stretching quadrant or the unstable node/saddle/saddle quadrant. Note that this visual preference is only referring to a rather weak occurrence. The reason is that the p.d.f. values are scaled logarithmically, meaning that the majority of points lie near the origin. The conditionally sampled particle p.d.f.s (figure $4 b, c$ ) show an almost similar behaviour for the inward (to the wall) migrating particles, whereas the outward (to the channel centre) migrating particles have apparently a slightly higher preference for the stable regions, i.e. $\operatorname{Re}\left(\lambda_{2}\right), \operatorname{Re}\left(\lambda_{3}\right)<0$ (topological quadrants II and III).

The two-phase coupling effect (due to the interaction between particles and turbulence) on the joint p.d.f.s of the flow become better revealed as figure $4(d, g)$ is considered. The following trend can be noticed: an increasing particle mass 
loading appears to significantly stretch the joint p.d.f.s along the $Q_{A}$-axis, while the distributions are (apart from the topological region $D_{A}<0$ and $R_{A}>0$ ) only modestly modified in the $R_{A}$-direction. This particle-induced intensifying behaviour of $Q_{A}$, without a similar amplification of the corresponding $R_{A}$ values, shows that the local flow topology becomes increasingly more two-dimensional. As was shown by many previous particle-laden studies (see for instance Rouson 1997; Yamamoto et al. 2001; Marchioli \& Soldati 2002; Portela \& Oliemans 2003), our particles also have a strong preference to accumulate in the low-speed streaks. The level of this particle accumulation behaviour controls the laminarization process of the near-wall turbulence. However, the instantaneous crossflow motions between low- and highspeed regions are not entirely annihilated. Due to the applied wall impermeability and continuity constraints, a kind of intense two-dimensional swirl occurs occasionally at, or near, the stronger contrasting interfaces of the particle-dictated low-speed streaks and flow-dominated high-speed regions. Since such wall-parallel crossflow swirls are typically characterized by high velocity-gradients, the $Q_{A}$ values will rise.

Finally, the two-way coupling effect on the joint p.d.f.s conditionally sampled at the particle positions (figure $4 b, c, e, f, h, i$ ) is essentially the same as for the one-way coupled simulation, although stronger for the inward-moving particles than for the outward moving ones. The $Q_{A}, R_{A}$ distribution of the particles migrating towards the wall $\left(W_{p}<0\right)$ shows a reasonable match with the more active turbulent regions of their corresponding distributions sampled at the grid points, whereas the invariant distributions for $W_{p}>0$ indicate that a larger fraction of the particles are preferentially staying in the more stable and less vortical/dissipative intense regions. Since larger excursions of the joint p.d.f.s at the inward-migrating particle locations appear to have a greater resemblance with the joint p.d.f.s at the grid points, it suggests that particularly these particles (which are smaller in number) have a strong influence on modifying the extremes of the local flow topology. This indicates that two modification processes are important: (i) the inward-moving particles are abruptly dumping their abundant streamwise momentum in the viscous sublayer and (ii) the more pronounced tear-drop shaped $Q_{A}, R_{A}$ distribution, which (as will be shown later) is clearly visible at larger distances from the wall, reveals that the inward-moving particles are efficiently transferring topological information from the buffer region towards the direct vicinity of the wall.

\subsection{Viscous sublayer (from an ordinary spatial perspective)}

As we have seen, some of the geometry and statistical properties of the local flow patterns can be studied using the joint probability distributions of the two invariants. However, they provide no information how the local flow topologies are actually grouped in ordinary physical space. To improve on this point we have calculated a number of instantaneous wall-parallel cross-sections of relevant flow quantities.

When first the particle and axial velocity distributions are considered (an example is given in figure 5), one can clearly see that the vast majority of the outward migrating particles are located in, or near, the low-speed streaks, whereas the inward (to the wall) migrating particles are more randomly distributed. The accumulation of particles at the wall leads to a laminarization of the near-wall turbulence.

We have made a more detailed study of the relation between particle concentration and streamwise velocity fluctuation as function of the distance to the wall. Figure 6 shows the global behaviour of the streamwise velocity fluctuation p.d.f.s as a normalized contour plot for all $z^{+}$in a half channel. When, first, the unconditional unladen $u^{\prime}$-distribution is considered (figure $6 a$ ), one can notice that the variance of 

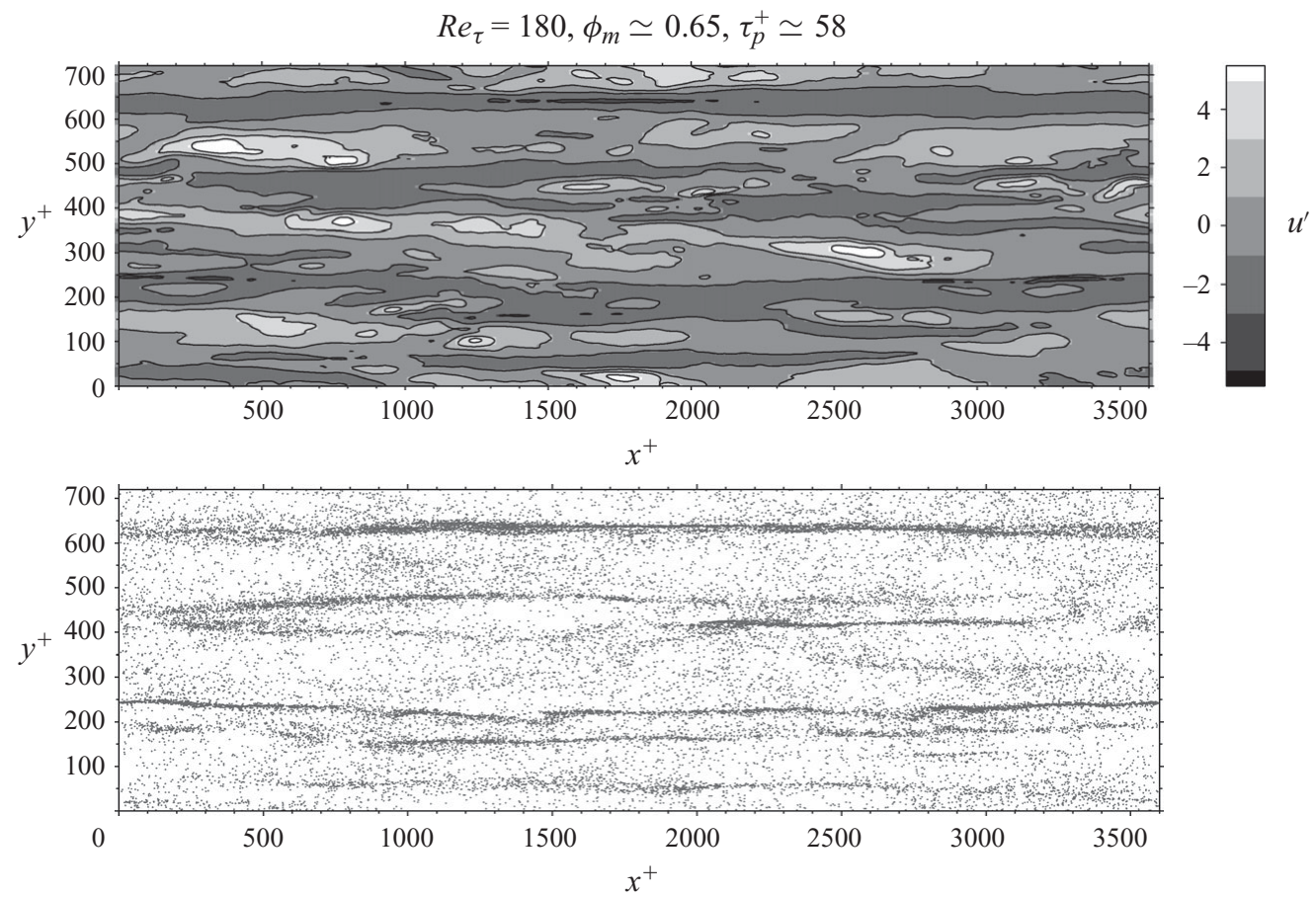

FIGURE 5. Instantaneous wall-parallel cross-sections of streamwise velocity fluctuations, and corresponding particle distributions, located at $z^{+} \simeq 5$.

$u^{\prime}$ becomes relatively large in the energetic buffer region, whereas it reduces to smaller values close to the wall (no-slip condition) and outer region of the channel. The buffer region is characterized by the violent, anisotropic character of the flow, generating most of its turbulent energy. Therefore, to be able to distinguish what part of the $u^{\prime}$-distribution is a result of in- or outrush of fluid motions, the total p.d.f. contour is divided into two conditional parts: (i) $u^{\prime}\left(w^{\prime}<0\right)$-distribution, figure $6(b)$ : streamwise turbulent motion, which is advected towards the wall and (ii) $u^{\prime}\left(w^{\prime}>0\right)$-distribution, figure 6(c): streamwise turbulent motion, which is advected towards the channel centre. These conditional distributions confirm that the streamwise turbulent fluctuations are about twice as likely to be negatively correlated than positively correlated with the wall-normal fluctuations, i.e. generating overall a negative turbulent shear stress.

The $u^{\prime}$-distributions of the high mass loading are displayed in figure $6(d-f)$. Here we see that the mass loading has only a small effect on increasing the variance of $u^{\prime}$. At first sight one may think that this small difference is negligible. However, recall that most of the turbulent energy is essentially located in the tails of a p.d.f. Figure $6(f)$ reveals that most of this enhancement is caused by the more pronounced increase of slow outward streamwise fluctuations (compared to figure $6 c$ ), and that the inrush of high streamwise momentum fluctuations remains more or less unaffected by the presence of the particles. To evaluate if this enhancement of the variance of $u^{\prime}$ is a consequence of the momentum exchange between particles and fluid, the $u^{\prime}$-distributions seen at the particle locations are given in figure $6(g-i)$. As can be seen in figure $6(i)$, most outward migrating particles within the near-wall region are segregated in the regions of lower-than-mean fluid velocity fluctuations, confirming, 

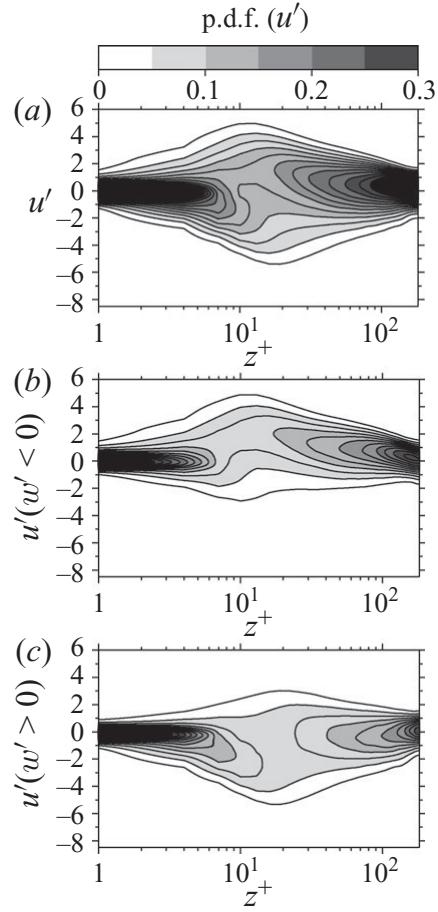
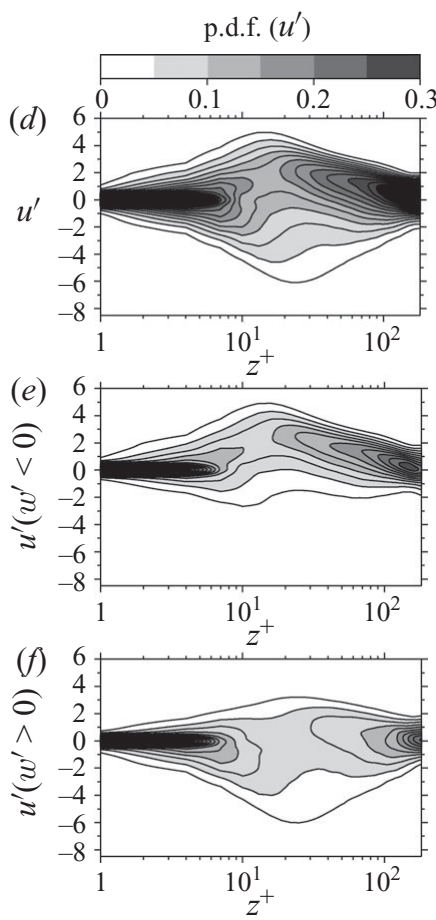
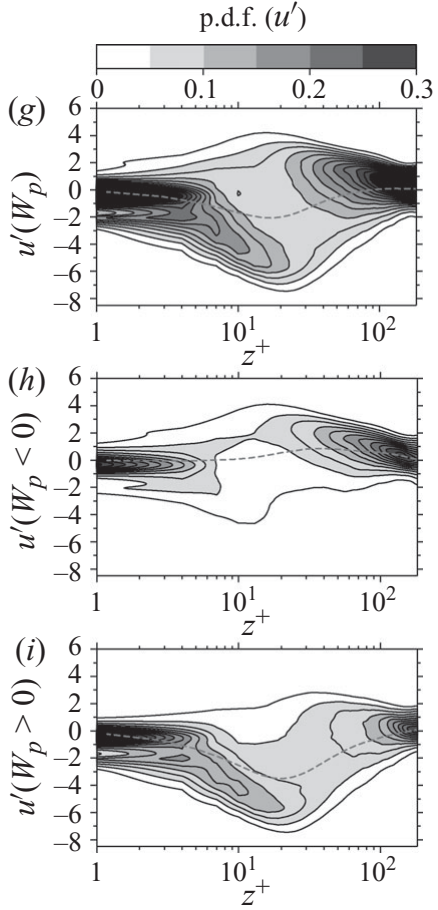

FIGURE 6. p.d.f. contours of streamwise velocity fluctuations, for $R e_{\tau}=180$ : $(a-c)$ unladen case; $(d-i) \phi_{m} \simeq 0.65, \tau_{p}^{+} \simeq 58$. $(a-f)$ display p.d.f. $\left(u^{\prime}\right)$ contours obtained from Eulerian field-description; $(g-i)$ p.d.f. $\left(u^{\prime}\right)$ contours obtained at the locations of the particles, where the average streamwise drift velocities $\left(\overline{u^{\prime}}\right)_{p}$ are shown as dashed lines. Note: for arbitrary fixed $z^{+}$, the total integral value of p.d.f. $\left(u^{\prime}\right)$ is normalized and equals unity, while the p.d.f. contours which make a distinction between wall- and centre-wards motions are normalized according to their share in constructing p.d.f. $\left(u^{\prime}\right)$, i.e. p.d.f. $\left(u^{\prime}\right)=$ p.d.f. $\left(u^{\prime}\left(w^{\prime}<0\right)\right)+$ p.d.f. $\left(u^{\prime}\left(w^{\prime}>0\right)\right)=1$, $\forall z^{+}$.

as was also observed by many others (see e.g. Wang \& Squires 1996; Rouson \& Eaton 2001), that the particles are preferentially located within the low-speed streaks. Unlike the outward migrating particles, which modify the near-wall streaky flow structures, the incoming particles $\left(W_{p}<0\right)$ have only a small positive streamwise drift-velocity, indicating a less pronounced tendency to segregate into specific flow regions. This difference in behaviour between in- and outward migrating particles shows that incoming particles are less effective in modifying 'directly' their local fluid surroundings, yielding a smaller pseudo-turbulence contribution than is found for the outward migrating particles.

Figures $7(a-c)$ and $8(a-c)$ depict the instantaneous distributions of $Q_{A}$ in the viscous sublayer at two distances from the wall. As can be seen, the distributions gain a much finer structure with increasing particle mass loading (particularly in the direct vicinity of the wall). To understand this change of $Q_{A}$ with mass loading more readily, we can alternatively express the second invariant through taking the divergence on the Navier-Stokes equations (multiplied by $-1 / 2$ ), leading to the two-way coupled Poisson equation for pressure

$$
Q_{A}=-\frac{1}{2} \frac{\partial U_{i}}{\partial x_{j}} \frac{\partial U_{j}}{\partial x_{i}}=\frac{1}{2} \frac{\partial^{2} P}{\partial x_{i}^{2}}-\frac{1}{2} \frac{\partial F_{i}}{\partial x_{i}} .
$$



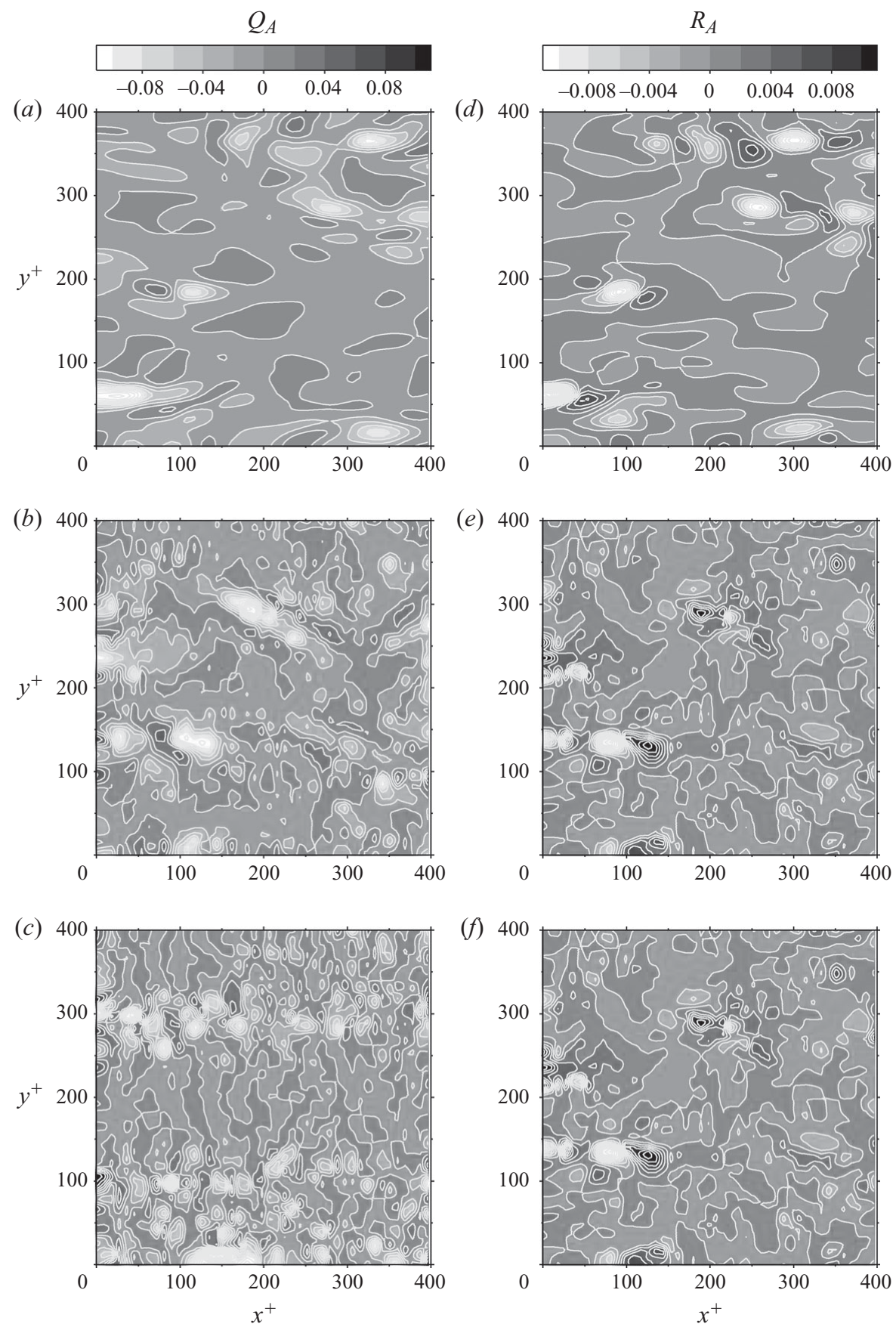

FIGURE 7. Effect of mass loading on instantaneous wall-parallel snapshots, located at $z^{+} \simeq 1$. Test cases $R e_{\tau}=180, \tau_{p}^{+} \simeq 58:(a)$ and $(d)$ O3.3: one-way coupling; (b) and $(e) \mathrm{R} 3.3: \phi_{m} \simeq 0.16$; (c) and $(f)$ R3.4: $\phi_{m} \simeq 0.65$. $(a-c) Q_{A} ;(d-f) R_{A}$.

From (4.1), it becomes clear that $Q_{A}$ reflects the difference between the pressure and 'direct' particle forcing terms. We found that an increasing mass loading has generally a smoothing effect on the pressure contribution (see Bijlard 2009). Therefore the 

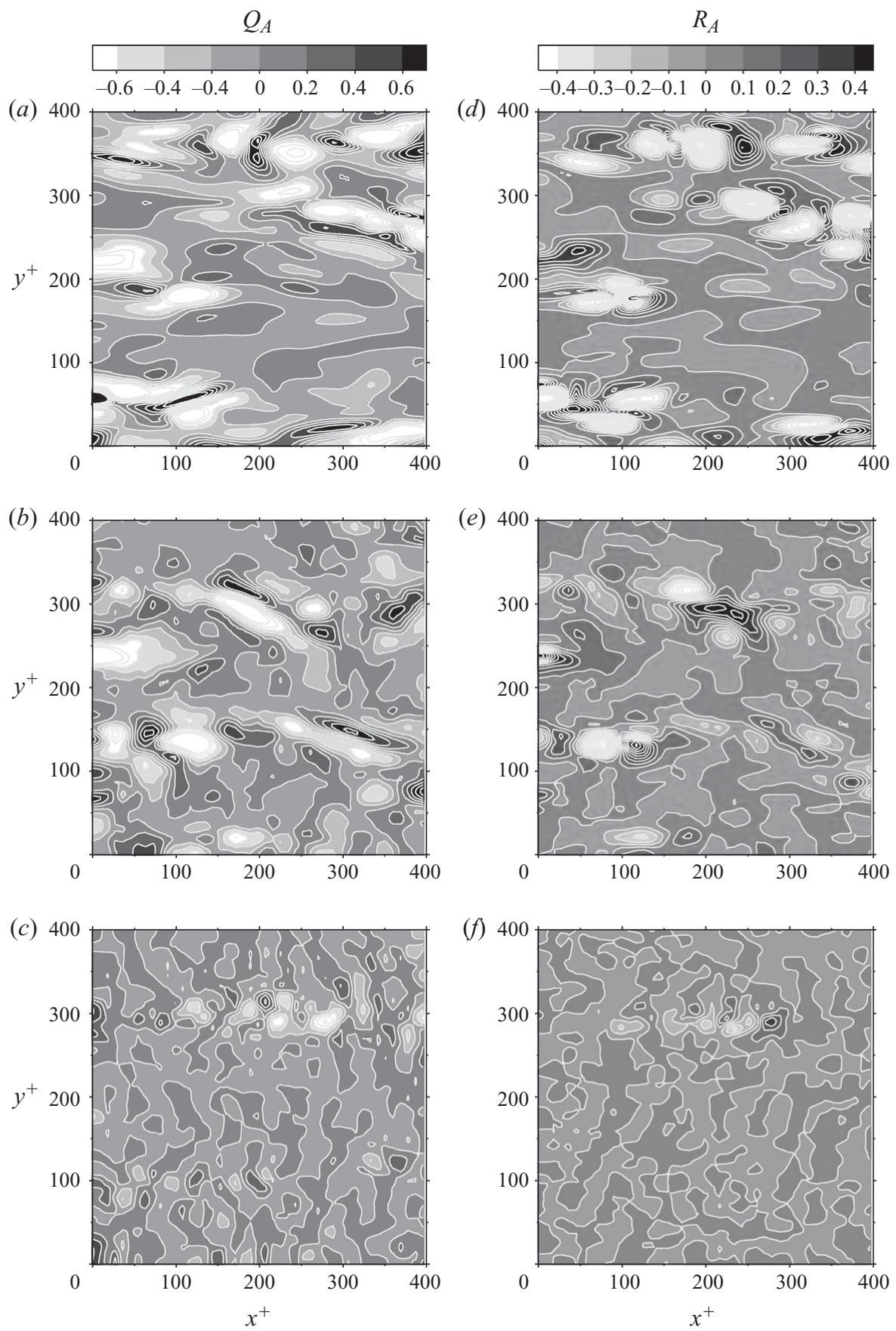

FIGURE 8. Effect of mass loading on instantaneous wall-parallel snapshots, located at $z^{+} \simeq 5$. Test cases $R e_{\tau}=180, \tau_{p}^{+} \simeq 58:(a)$ and $(d)$ O3.3: one-way coupling; $(b)$ and $(e) \mathrm{R} 3.3: \phi_{m} \simeq 0.16$; (c) and $(f) \mathrm{R} 3.4: \phi_{m} \simeq 0.65$. $(a-c) Q_{A} ;(d-f) R_{A}$.

observed modifications in $Q_{A}$ are probably the result of the local forcing term. Inward-migrating particles are more uniformly distributed and experience relatively large slip velocities. Outward migrating particles are, on the other hand, larger 
in number but prefer to accumulate in the low-speed regions. Due to this massive collective behaviour of the outward migrating particles the dynamics of the low-speed regions become increasingly dictated by the dispersed phase. Since the distribution of $Q_{A}$ gains a much finer structure with increasing particle mass loading, it indicates that particularly the inward-migrating particles are better capable of disrupting the local turbulent velocity field, i.e. producing, on average, much larger velocity gradients than the outward migrating particles.

The corresponding instantaneous distributions of $R_{A}$ are displayed in figures $7(d-f)$ and $8(d-f)$. The figures show that in the direct vicinity of the wall $\left(z^{+} \simeq 1\right)$, the value of $R_{A}$ does not change much with increasing mass loading. However, at larger distances from the wall $\left(z^{+} \simeq 5\right)$, this value decreases strongly with increasing mass loading. This is consistent with the enhanced laminarization effect with increasing $\phi_{m}$. Indeed for the one-way coupled simulation (figures $7 d$ and $8 d$ ), the intense vortex-stretching regions $\left(R_{A}<0\right)$ become progressively more developed at greater distances from the wall, i.e. stronger, elongated and parallel with the flow direction (similar results were obtained by Blackburn et al. (1996). However, this does not hold anymore as the mass loading becomes significant (figures $7 f$ and $8 f$ ). This indicates that any intense vortex-stretching region becomes essentially annihilated by the presence of the particles. Combining the observations of $Q_{A}$ and $R_{A}$ shows that the small-scale dynamical modifications in the viscous sublayer are a result of the local energy exchange of the particles with their surrounding fluid. Our results for $Q_{A}$ and $R_{A}$ reveal clearly that an increasing particle mass loading suppresses new born vortex-stretching regions in the viscous sublayer by fragmenting them, leading to smaller structures that become naturally exposed to the final molecular destruction process.

\subsection{Buffer region}

Figure 9 shows again the volume-averaged invariant distributions in the buffer region $\left(5<z^{+} \simeq 35\right)$ for the one-way coupling and two-way coupling cases for two different mass loadings. When, first, the one-way coupled joint p.d.f. is considered (figure $9 a$ ), one can notice (in agreement with other wall-bounded studies, see Blackburn et al. 1996 and Rouson \& Eaton 2001) that: (i) within the buffer region the velocitygradient contributions are significantly larger than in the viscous sublayer and (ii) the maximum values of the invariants have an even more pronounced preference for the second and fourth topological quadrants. The corresponding joint p.d.f.s conditionally sampled at the particle locations (figure $9 b, c$ ) do not show any significant differences compared with the distribution at the grid points. This behaviour is consistent with the results of Rouson \& Eaton (2001).

The effect of two-way coupling due to an increasing mass loading reveals that the addition of particles reduces considerably the size of the invariant distributions (figure $9 a \rightarrow d \rightarrow g$ ). As can be noticed in figure 9 the joint p.d.f.s of the fluid at the particle locations are practically identical with the corresponding distributions of the fluid at the grid points. This reduction in size of the invariant distribution in the buffer region is caused by the strong laminarization effect of the particles on the viscous sublayer.

\subsection{Logarithmic layer}

Figure 10 shows the two-way coupling effect on the joint p.d.f.s in the logarithmic layer $\left(35<z^{+} \lesssim 150\right)$. As can be noticed, the shape of the local flow topology distributions has a strong resemblance with the ones observed in the buffer region. It was also found that the intermediate inertia particles $\left(\tau_{p}^{+} \simeq 28\right.$ and $\left.\tau_{p}^{+} \simeq 58\right)$ have a stronger 

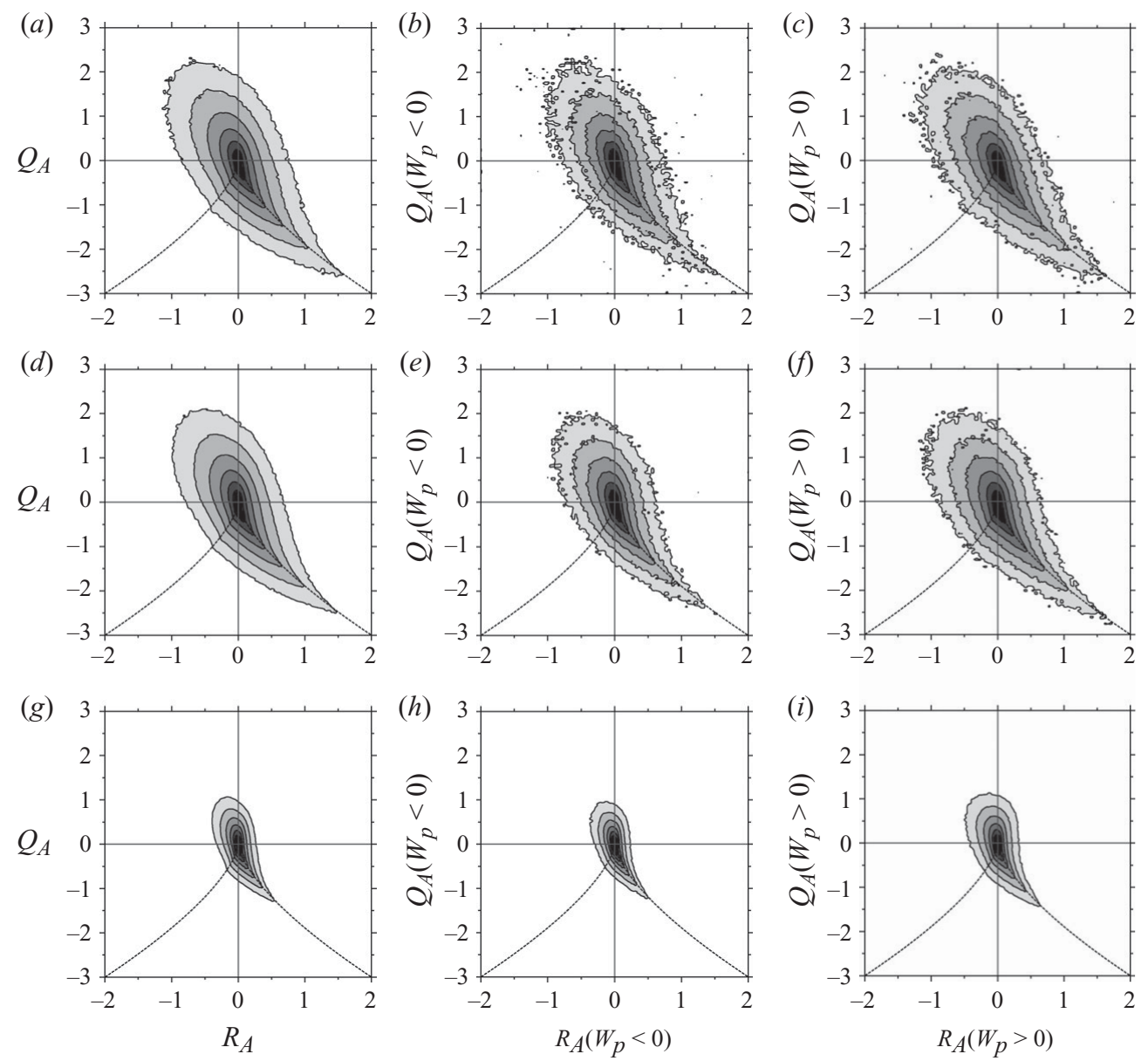

FIGURE 9. Particle mass loading effect on the $Q_{A}-R_{A}$ plane, for the buffer region $\left(5<z^{+} \leqslant 35\right)$. Similar notation has been used as figure 4 .

suppressing effect on the invariant distributions than the lighter and heavier particles. This is due to two effects, namely: (i) these particles segregate preferentially in the low-speed streaks and (ii) the acceleration of these particles, when migrating outwards, is on average relatively slow. At large wall distances they still withdraw a significant amount of momentum from their surrounding fluid. The joint p.d.f.s sampled conditionally at the particle locations $\left(W_{p} \lessgtr 0\right)$ indicate that the outward migrating particles preferentially stay in more active turbulent regions, while the inward-migrating particles show an opposite trend.

Wall-parallel instantaneous snapshots for $Q_{A}$ and $R_{A}$ are shown in figure 11. As can be seen the particles have a strong damping effect on these quantities (as found at $\left.z^{+}=5\right)$.

\subsection{Outer region}

Figures 12 and 13 show the two-way coupling effect on the joint p.d.f.s in the outer region of the channel $\left(150<z^{+}<R e_{\tau}\right)$. Like the adjacent channel regions we notice that the turbulence activity reduces as $\phi_{m}$ increases. The shape of the local flow topology remains approximately maintained, indicating that the turbulence structures 

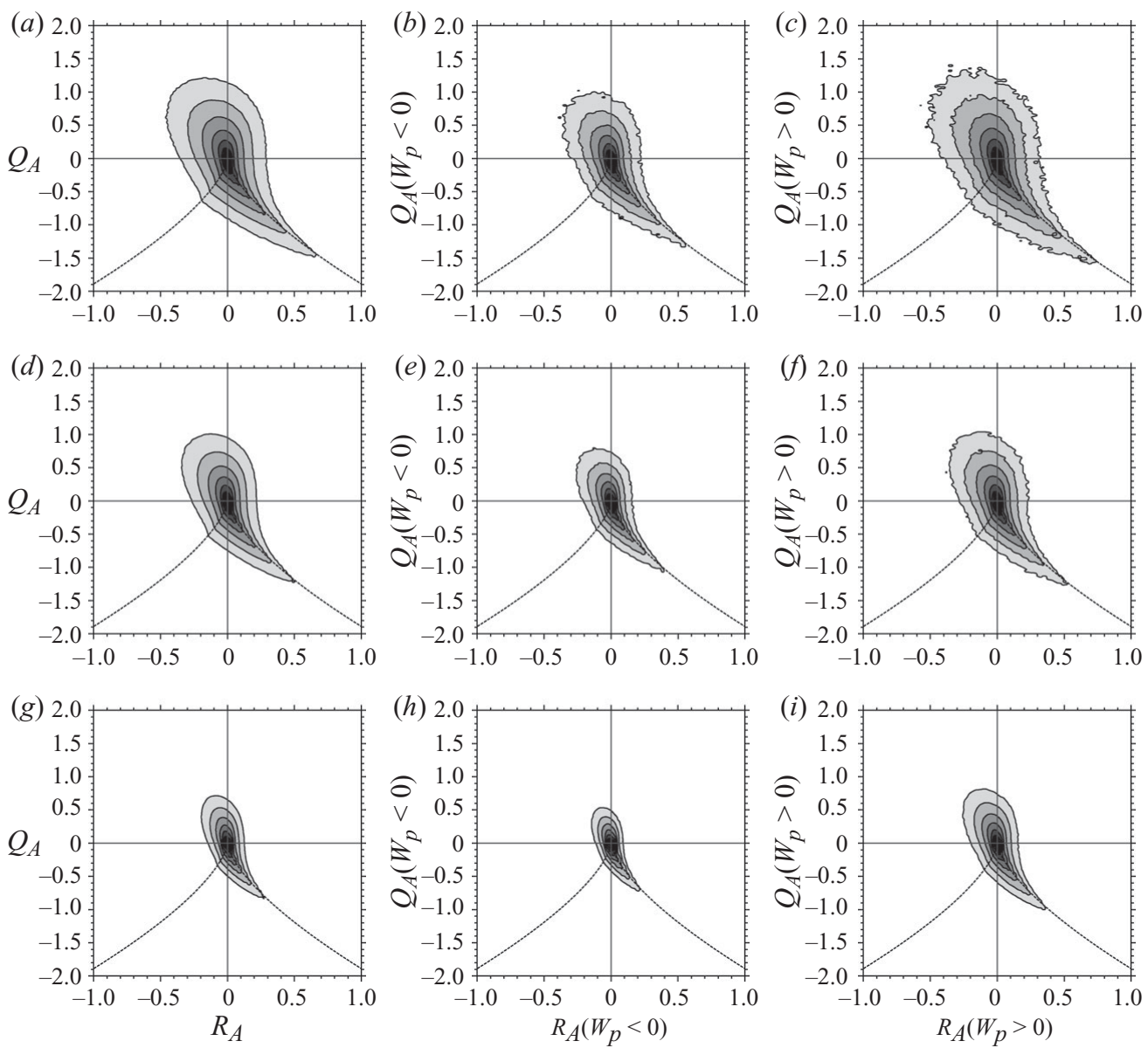

Figure 10. Particle mass loading effect on the $Q_{A}-R_{A}$ plane, for the 'logarithmic' region $\left(35<z^{+} \leqslant 150\right)$. Similar notation has been used as figure 4 .

undergo a fairly uniform damping. It should be emphasized that this uniform damping is not directly caused by the presence of the particles; because (i) most turbulent energy is transported from the strongly modified wall region towards the centre region (in the centre region the production of turbulence is very small), (ii) the particle concentration is very dilute and (iii) the particle-fluid momentum exchange is small-to-modest.

The joint p.d.f.s sampled conditionally at the particle positions (figures $12 d-f$ and $13 d-f$ ) show, regardless of the two-way coupling mechanism, that the particles are preferentially located in less active turbulent regions. Furthermore, the distributions of the $\tau_{p}^{+} \simeq 58$ inertia particles are similar but smaller in size than their corresponding distributions sampled at the grid points. The local flow topology seen by lighter inertia particles $\tau_{p}^{+} \simeq 14$ and $\tau_{p}^{+} \simeq 28$, however, do not show such uniform decrease in size. These particles have a stronger tendency to be located in the fourth topological quadrant. These findings are in contrast with the one-way coupling results reported by Rouson \& Eaton (2001). They found essentially no $S t$-dependent differences.

A better agreement is found when we compare our results with the forced-isotropic particle-laden turbulence study performed by Squires \& Eaton (1991). In their modest parameter study (containing three Stokes numbers: $S t_{K} \simeq 0.325 / 0.65 / 2.27$ ), 

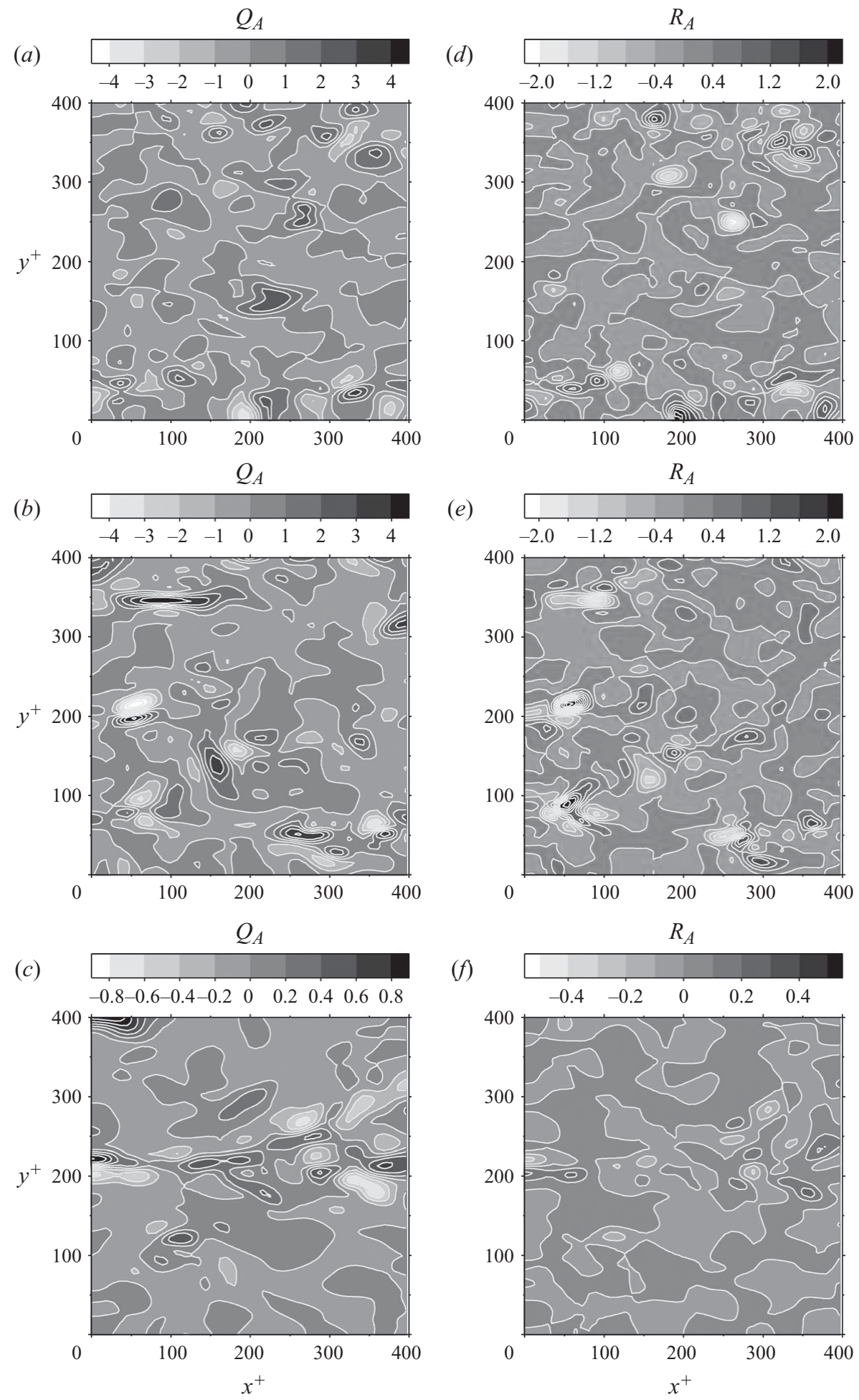

FIGURE 11. Instantaneous snapshots of $Q_{A}$ and $R_{A}$ corresponding to figure 10. Similar notation used as figure 7. 

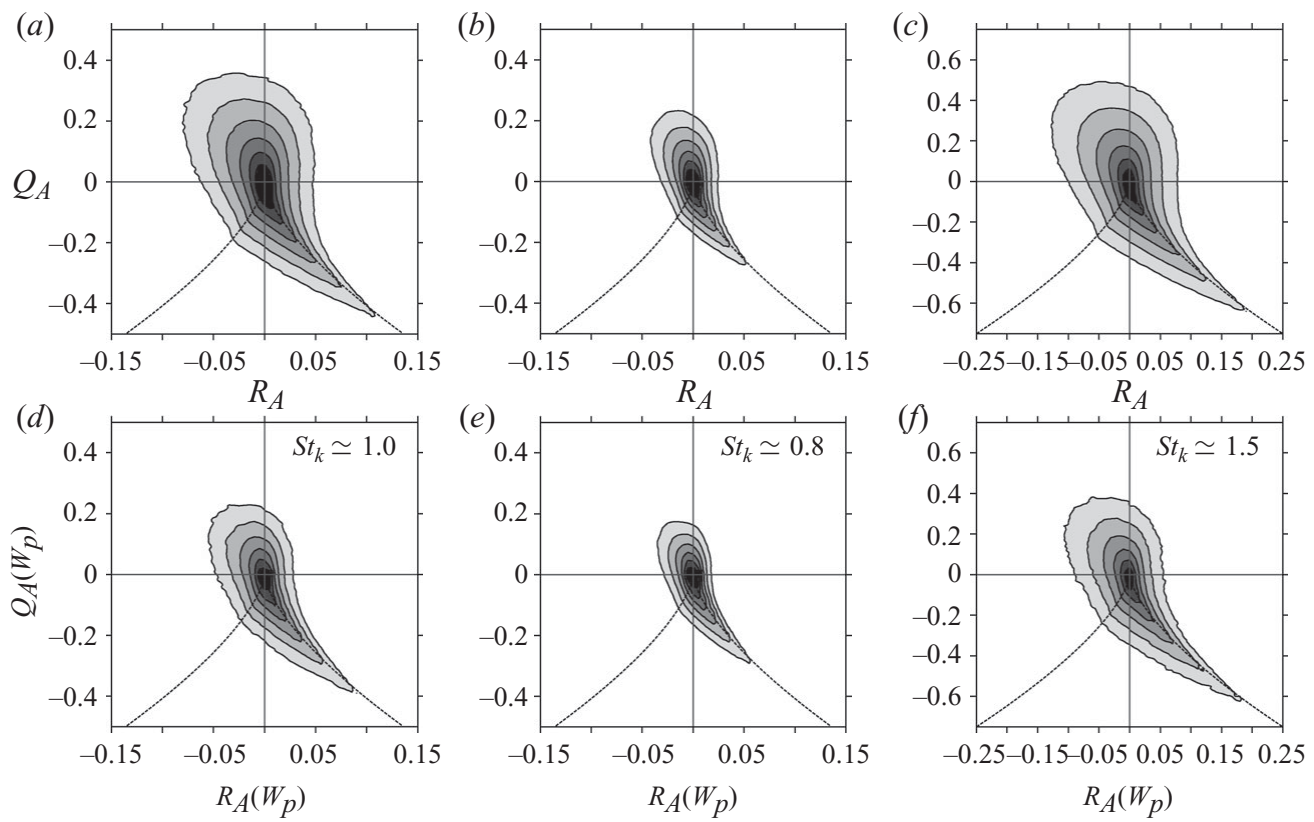

Figure 12. Two-way coupling effects on normalized joint p.d.f. of $Q_{A}, R_{A}$, in the outer region $150<z^{+}<R e_{\tau} .(a-c)$ sampled at the grid locations; $(d-f)$ sampled at particle locations. $(a)$ and (d) R3.1: $R e_{\tau}=180, \phi_{m} \simeq 0.16, \tau_{p}^{+} \simeq 14 ;(b)$ and $(e) \mathrm{R} 3.3: \operatorname{Re}_{\tau}=180, \phi_{m} \simeq 0.65, \tau_{p}^{+} \simeq 14$; (c) and $(f) \mathrm{R} 5.1: R e_{\tau}=250, \phi_{m} \simeq 0.16, \tau_{p}^{+} \simeq 28$. The grey scales denote a logarithmic decade, ranging over six decades.
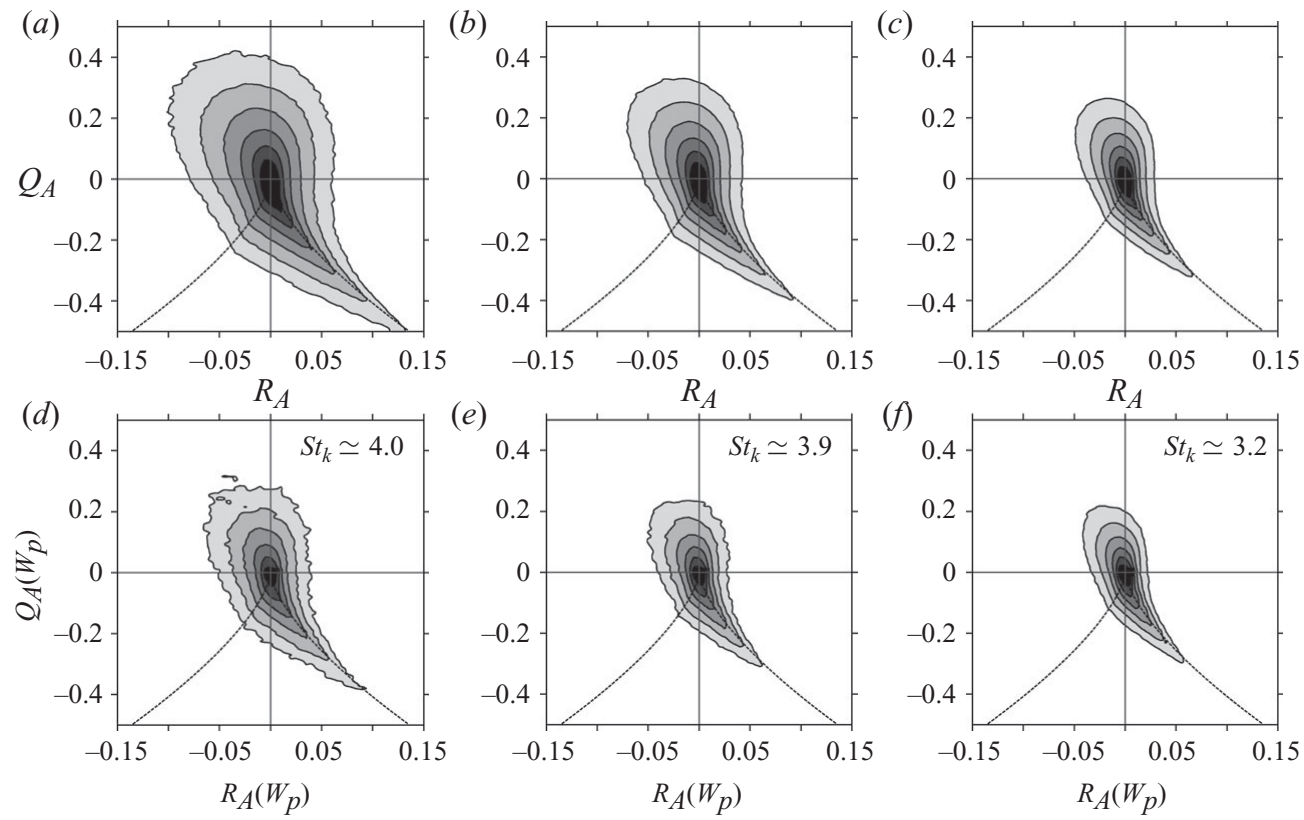

FIGURE 13. Two-way coupling effects on normalized joint p.d.f. of $Q_{A}, R_{A}$, in the outer region $150<z^{+}<R e_{\tau} .(a-c)$ sampled at the grid locations; $(d-f)$ sampled at particle locations. (a) and $(d)$ O3.3: one-way coupling; $(b)$ and $(e) \mathrm{R} 3.3: \phi_{m} \simeq 0.16, \tau_{p}^{+} \simeq 58 ;(c)$ and $(f) \mathrm{R} 3.4$ : $\phi_{m} \simeq 0.65, \tau_{p}^{+} \simeq 58$. The grey scales denote a logarithmic decade, ranging over six decades. 
they reported that particularly the particles with an inertia of $S t_{K} \simeq 0.65$ cause a significant bias in trajectory towards regions of low vorticity and high strain rate, i.e. $Q_{A}<0$. A straightforward comparison is, however, rather difficult to make, mainly because (i) our centreplane statistics cannot be treated as isotropic and (ii) their topology study is based on a somewhat older classification scheme (see Wray \& Hunt 1989) that uses only the second invariant plus some additional, ambiguous thresholds). Despite these differences our result for the light particles $S t_{K} \simeq[0.8-1.5]$ supports their findings, and moreover, the well-accepted physical ideas that particularly these kind of inertia particles spiral away from vortex cores due to centrifugal effects, while lighter or heavier inertia particles give rise to a more random behaviour. Additional to their, and many other, particle-laden studies is that our invariant distributions may suggest that light particles with $S t_{K} \approx 1.0$ have a tendency to accumulate near vortex sheets. The reason is that the fourth topological quadrant corresponds to local flow topologies where biaxial strain is dominant, and this is commonly associated with vortex-sheet formation (see Batchelor 1953).

\section{Conclusion}

In the viscous sublayer the local flow topology becomes increasingly more twodimensional, switching between either strong vortical motions or strong strain regions, as two-way coupling effects increases. This highly intermittent small-scale behaviour is strongly associated with the large particle accumulation into the elongated streaks near the wall, enlarging the contrasts between the streamwise-elongated low- and high-speed regions of the flow. This streaky particle segregation causes an increasing annihilation of the intense vortex-stretching regions by 'prematurely' fragmenting them; hence preventing a normal development of near-wall turbulence. Beyond the viscous sublayer the modifications in the invariant maps do not appear to be strongly related with the preferential concentration of the particles, which is in contrast to previous channel flow simulations. The reducing effect on the invariant distributions of the fluid by the particles is merely reflecting the indirect effects of the overall particle-turbulence interaction, and is mostly a result of the overall changing nearwall dynamics.

\section{REFERENCES}

Ahmed, A. M. \& Elghobashi, S. 2000 On the mechanisms of modifying the structure of turbulent homogeneous shear flow by dispersed particles. Phys. Fluids 12 (11), 2906-2930.

Batchelor, G. K. 1953 The Theory of Homogeneous Turbulence. Cambridge University Press.

BiJLARD, M. J. 2009 Analysis of particle-induced turbulence modulation and two-fluid closures by DNS channel flow. PhD thesis, Delft University of Technology, Delft, the Netherlands.

Blackburn, H. M., Mansour, N. N. \& Cantwell, B. J. 1996 Topology of fine-scale motions in turbulent channel flow. J. Fluid Mech. 310, 269-292.

Boivin, M., Simonin, O. \& Squires, K. D. 1998 Direct numerical simulation of turbulence modulation by particles in isotropic turbulence. J. Fluid Mech. 375, 235-263.

Chacín, J. M. \& Cantwell, B. J. 2000 Dynamics of a low-Reynolds-number turbulent boundary layer. J. Fluid Mech. 404, 87-115.

Chacín, J. M., Cantwell, B. J. \& Kline, S. J. 1996 Study of turbulent boundary layer structure using the invariants of the velocity gradient tensor. Exp. Therm. Fluid Sci. 13, 308-317.

Chong, M. S., Perry, A. E. \& Cantwell, B. J. 1990 A general classification of three-dimensional flow fields. Phys. Fluids A 2 (5), 765-777.

Elghobashi, S. 1994 On predicting particle-laden turbulent flows. Appl. Sci. Res. 52, 309-329. 
Elghobashi, S. \& Truesdell, G. C. 1993 On the two-way interaction between homogeneous turbulence and dispersed solid particles. Part I. Turbulence modification. Phys. Fluids A 5 (7), 1790-1801.

Li, Y., Mclaughlin, J. B., Kontomaris, K. \& Portela, L. M. 2001 Numerical simulation of particle-laden turbulent channel flow. Phys. Fluids 13 (10), 2957-2967.

Marchioli, C. \& Soldati, A. 2002 Mechanisms for particle transfer and segregation in a turbulent boundary layer. J. Fluid Mech. 468, 283-315.

Marchioli, C., Soldati, A., Kuerten, J. G. M., Arcen, B., Taniere, A., Goldensoph, G., Squires, K. D., Cargnelutti, L. M. \& Portela, L. M. 2008 Statistics of particle dispersion in direct numerical simulations of wall-bounded turbulence: results of an international collaborative benchmark test. Intl J. Multiphase Flow 34, 879-893.

Ooi, A., Martin, J., Soria, J. \& ChOng, M. S. 1999 A study of the evolution and characteristics of the invariants of the velocity-gradient tensor in isotropic turbulence. J. Fluid Mech. 381, $141-174$.

Perry, A. E. \& ChOng, M. S. 1994 Topology of flow patterns in vortex motions and turbulence. Appl. Sci. Res. 53, 357-374.

Picciotto, M., Marchioli, C. \& Soldati, A. 2005 Characterization of near-wall accumulation regions for inertial particles in turbulent boundary layers. Phys. Fluids 17, 098101.

Portela, L. M. \& Oliemans, R. V. A. 2003 Eulerian-Lagrangian DNS/LES of particle-turbulence interactions in wall-bounded flows. Intl J. Numer. Methods Fluids 43, 1045-1065.

Rouson, D. W. I. 1997 A direct numerical simulation of a particle-laden turbulent channel flow. PhD thesis, Mechanical Engineering Department, Stanford University, Palo Alto, California.

Rouson, D. W. I. \& EATON, J. K. 2001 On the preferential concentration of solid particles in turbulent channel flow. J. Fluid Mech. 428, 149-169.

Soldati, A. 2005 Particles turbulence interactions in boundary layers. Z. Angew. Math. Mech. 85 (10), 683-699.

Soria, J. \& CANTwell, B. J. 1994 Topological visualization of focal structures in free shear flows. Appl. Sci. Res. 53, 375-386.

Soria, J., OoI, A. \& ChOng, M. S. 1997 Volume integrals of the $Q_{A}-R_{A}$ invariants of the velocity gradient tensor in incompressible flows. Fluid Dyn. Res. 19, 219-233.

Soria, J., Sondergatrd, R., Cantwell, B. J., Chong, M. S. \& Perry, A. E. 1994 A study of finescale motions of incompressible time-developing mixing layers. Phys. Fluids 6 (2), 871-884.

Squires, K. D. \& Eaton, J. K. 1990 Particle response and turbulence modification in isotropic turbulence. Phys. Fluids A 2 (7).

Squires, K. D. \& Eaton, J. K. 1991 Preferential concentration of particles by turbulence. Phys. Fluids A 3 (5), 1169-1178.

Tennekes, H. \& Lumley, J. L. 1972 A First Course in Turbulence. MIT Press.

Uhlmann, M. 2008 Interface-resolved direct numerical simulation of vertical particulate channel flow in the turbulent regime. Phys. Fluids 20 (053305).

WANG, Q. \& SQuires, K. D. 1996 Large eddy simulation of particle-laden turbulent channel flow. Phys. Fluids 8 (5), 1207-1223.

Wray, A. A. \& Hunt, J. C. R. 1989 Algorithms for classification of turbulent structures. In Topological Fluid Mechanics; Proceedings of the IUTAM Topology of Fluid Mechanics, Cambridge, UK, 13-18 August 1989 (ed. H. K. Moffat), pp. 95-104. Cambridge University Press.

Yamamoto, Y., Potthoff, M., Tanaka, T., Kajishima, T. \& Tsuji, Y. 2001 Large-eddy simulation of turbulent gas-particle flow in a vertical channel: effect of considering inter-particle collisions. J. Fluid Mech. 442, 303-334. 